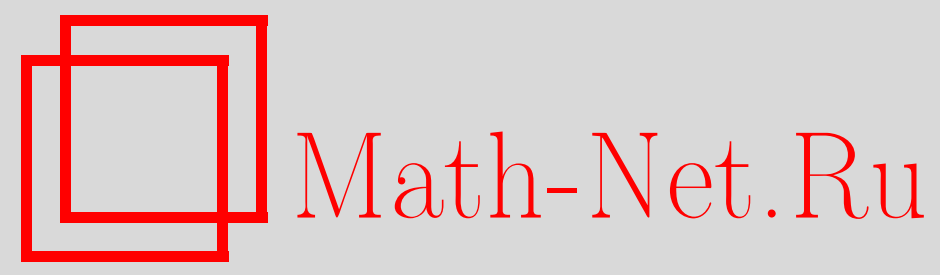

М. С. Сгибнев, О распределении супремума случайного блуждания при наличии корней характеристического уравнения, Теория вероятн. и ее примен., 1998, том 43, выпуск $2,383-390$

DOI: https://doi.org/10.4213/tvp1475

Использование Общероссийского математического портала Math-Net.Ru подразумевает, что вы прочитали и согласны с пользовательским соглашением http://www . mathnet.ru/rus/agreement

Параметры загрузки:

IP : 54.174 .149 .18

26 апреля 2023 г., 18:34:26

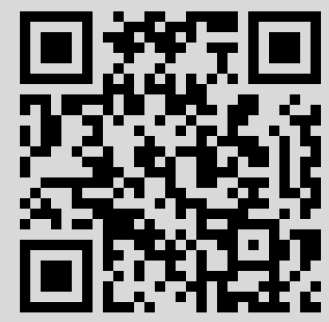


(c) $1998 \mathrm{r}$.

СГИБНЕВ M. C.*

\section{О РАСПРЕДЕЛЕНИИ СУПРЕМУМА СЛУЧАЙНОГО БЛУЖДАНИЯ ПРИ НАЛИЧИИ КОРНЕЙ ХАРАКТЕРИСТИЧЕСКОГО УРАВНЕНИЯ ${ }^{1}$}

Рассматривается случайное блуждание $\left\{S_{n}\right\}$, порожденное последонательностью $\left\{X_{k}\right\}$ независимых одинахово распределенных случайных величин с $\mathbf{E} X_{1} \in$ $(-\infty, 0)$. Исследуется влияние корней характеристического уравнения 1 $\mathbf{E} \exp \left(s X_{1}\right)=0$ в полосе аналитичности преобразования Лапласа $\mathbf{E} \exp \left(s X_{1}\right)$ на распределение супремума $\sup _{n} \geqslant 0 S_{n}$. Аналогичная задача решается и для стационарного распределения осциллирующего случайного блуждания.

Каючевье слова и Фразы: случайное блуждание, супремум, корни харахтеристического уравнения, абсолютно непрерывная компонента, осциллирующее случайное блуждание, стащионарное распределение, асимптотическое поведение.

1. Введение. Пусть $\left\{X_{k}\right\}_{k=1}^{\infty}$ - последовательность независимых одинаково распределенных случайных величин с распределением $F$ и математическим ожиданием $\mu=\mathbf{E} X_{1} \in(-\infty, 0)$. Обозначим $S_{0}=0, S_{n}=X_{1}+X_{2}+\cdots+X_{n}, n \geqslant 1$. Рассмотрим случайную величину $M_{\infty} \stackrel{\text { def }}{=} \sup _{n \geqslant 0} S_{n}$. С вероятностью единица $M_{\infty}<\infty$ $[1$, гл. XII, § 7].

Свойства распределения супремума $M_{\infty}$ изучались многими авторами. Так, в работах [2]-[6] рассматривался вопрос о существовании моментов $\mathbf{E} f\left(M_{\infty}\right)$ при определенном выборе функции $f(x)$, а именно: при степенных функциях, правильно менякщихся на бесконечности функциях, полумультипликативных функциях и др.; причем в отношении работы [4] следует отметить, что в [4, теорема 5] речь идет о моментах вида $\int f(x) \pi(d x)$ стационарного распределения $\pi$ цепи Маркова $Z_{n+1}=\max \left(Z_{n}+X_{n+1}, 0\right)$, однако хорошо известно [1, $\$$ VI.9.и $\S$ XVIII.5], что $\pi$ совпадает с распределением супремума $M_{\infty}$. В работах [7]-[13] основной акцент при изучени распределения супремума ставился на исследовании асимптотического поведения вероятности $\mathbf{P}\left\{M_{\infty}>x\right\}$.

В настоящей работе речь пойдет о распределении супремума $M_{\infty}$ при выполнении перечисленных ниже условий. Распределение супремума $M_{\infty}$ в виде сверточного сомножителя входит в выражения для стационарных распределений осциляируючего и почти однородного случайных блужданий [13]. Это обстоятельство позволяет применить методику данной работы к изучению стационарных распределений указанных марковских случайных блужданий. Мы ограничимся лишь случаем осциллируюшего случайного блуждания.

Основные предположения на распределение $F$, используемые в данной работе, таковы:

(a) преобразование Лапласа $\widehat{F}(s) \stackrel{\text { def }}{=} \int_{\mathbf{R}} \exp (s x) F(d x)$ определено в полосе П $(\tau)=$ $\{s \in \mathbf{C}: 0 \leqslant \operatorname{Re} s \leqslant r\}$ при некотором $r>0: \widehat{F}(r)<\infty$;

(б) характеристическое уравнение $1-\widehat{F}(s)=0$ имеет в полосе $\Pi(r)$ корни, отличные от нуля;

\footnotetext{
Россия.

1) Работа выполнена при поддержке Российского фонда фундаментальных исследований, код проекта 96-01-01939.
}

*Институт математики СО РАН, Университетский пр. 4, 630090 Новосибирск, 
(в) при некотором целом $m \geqslant 1$ распределение $F^{m *}$ ( $m$-кратная свертка распределения $F$ ) обладает ненулевой абсолютно непрерывной компонентой относительно меры Лебега.

Абсолютно непрерывную компоненту произвольного распределения $G$ обозначим через $G_{c}$, а его сингулярную компоненту - через $G_{s}: G_{s}=G-G_{c}$.

Допустим, что $\widehat{F}(s) \neq 1$ на прямой $\{s \in \mathbf{C}: \operatorname{Re} s=r\}$ и преобразование Лапласа в точке $r$ сингулярной компоненты $\left(F^{m *}\right)_{s}$ свертки $F^{m *}$ меньше единицы: $\left(F^{m *}\right)_{s}(r)<1$. Тогда в силу аналитичности функции $\hat{F}(s)$ в полосе П $(r)$ имеется лишь конечное число корней характеристического уравнения [14, теорема 3], причем среди них только один - вещественный, который мы обозначим через $q: q \in(0, r]$. Пусть $\mathscr{Z}=\{s \in \Pi(r): \widehat{F}(s)=1\} \backslash\{0\}$. Обозначим элементы множества $\mathscr{Z}$ через $q, s_{1}, s_{2}, \ldots, s_{l}$, а кратность корня $s_{j}$ - через $m_{j}$; это означает, что $1-\widehat{F}(s)=$ $\left(s-s_{j}\right)^{m} ; F_{j}(s)$, где $F_{j}\left(s_{j}\right) \neq 0$. Если $s \in \mathscr{Z}$ и $s \neq q$, то $\bar{s} \in \mathscr{Z}$ и корень $\bar{s}$ имеет ту же кратность, что и $s ;$ кроме того, $\operatorname{Im} s \neq 0$.

Пусть $\varphi(x), x \in \mathbf{R},-$ конечная положительная измеримая по Борелю функция, удовлетворяющая условиям $\varphi(0)=1, \varphi(x+y) \leqslant \varphi(x) \varphi(y), x, y \in \mathbf{R}$. Обозначим через $S(\varphi)$ совокупность всех комплекснозначных $\sigma$-конечных мер $\nu$ таких, что $\|\nu\|_{\varphi}=\int_{\mathbf{R}} \varphi(x)|\nu|(d x)<\infty$. Совокупность $S(\varphi)$ - банахова алгебра с нормой $\|\nu\|_{\varphi} ;$ операцией умножения элементов $\nu$ и $\kappa$ из $S(\varphi)$ служит их свертка $\nu * \kappa$, а единицей мера Дирака $\delta$. Теория банаховых алгебр $S(\varphi)$ хорошо изложена в [15, гл. 4] (правда, там рассматриваются банаховы алгебры мер, сосредоточенные на $[0, \infty)$, а не на всей прямой $\mathbf{R}$, но это различие несущественно).

Обозначим через $\mathfrak{S}_{r}$ банахову алгебру $S(\varphi)$ при следующем выборе полумультипликативной функции $\varphi(x): \varphi(x)=\exp (r x)$ при $x \geqslant 0$ к $\varphi(x) \equiv 1$ при $x<0$.

Определим оператор $T$ на множестве конечных мер $\kappa$. Будем обозначать функцию распределения конечной меры $\kappa$ той же самой буквой: $\kappa(x)=\kappa((-\infty, x])$. Положим $T(\kappa)(A)=\int_{A}(\delta(x)-\kappa(x)) d x$ (напомним: $\delta$ - мера Дирака, сосредоточенная в нуле). Если $\int_{\mathbf{R}}|x||\kappa|(d x)<\infty$, то $T(\kappa)-$ конечная мера. Кроме того, $T(\widehat{\kappa})(s)=(\widehat{\kappa}(s)-\widehat{\kappa}(0)) / s, \operatorname{Re} s=0$. Далее, если $\widehat{F}(r)<\infty$ для некоторого $r>0$, To $\int_{0}^{\infty} e^{r x}|T(F)|(d x)<\infty$.

2. Основной результат. Обозначим $D(x)=\mathbf{P}\left\{M_{\infty} \leqslant x\right\}$. Для преобразования Лапласа распределения $D$ справедливо представление [1, гл. XVIII, § 5, теорема 2]

$$
\widehat{D}(s)=\exp \left\{\sum_{n=1}^{\infty} \frac{1}{n} \int_{0-}^{\infty}\left(e^{s x}-1\right) F^{n *}(d x)\right\}, \quad \operatorname{Re} s \leqslant 0 .
$$

Обозначим через $G(x)$ функцию распределения первой отрицательной суммы $S_{N-\text {, }}$ $N-\stackrel{\text { def }}{=} \inf \left\{k \geqslant 1: S_{k}<0\right\}$. Тогда $[1$, гл. XVIII, §3, лемма 1]

$$
1-\widehat{G}(s)=\exp \left\{-\sum_{n=1}^{\infty} \frac{1}{n} \int_{-\infty}^{0-} e^{s x} F^{n *}(d x)\right\}, \quad \operatorname{Re} s \geqslant 0 .
$$

Положим $a=\exp \left\{\sum_{n=1}^{\infty} \mathbf{P}\left\{S_{n} \geqslant 0\right\} / n\right\}$. По известной теореме о факторизации [1, гл. XVIII, § 3] имеем

$$
1-\widehat{F}(s)=[a \widehat{D}(s)]^{-1} \cdot[1-\widehat{G}(s)], \quad \text { Re } s=0
$$

или

$$
\widehat{D}(s)=\frac{1-\widehat{G}(s)}{a[1-\widehat{F}(s)]}, \quad \operatorname{Re} s=0, s \neq 0 .
$$

Отношение, стоящее в правой части (2), представляет собой аналитическую функцию во внутренности полосы $\Pi(r)$, причем точки $q$ и $s_{j}$ суть изолированные особые точки 
этой функции, являющиеся полюсами порядка 1 и $m_{j}$ соответственно, $j=1, \ldots, l$. Пусть $\sum_{k=1}^{m_{j}}(-1)^{k} B_{j k} /\left(s-s_{j}\right)^{k}$ - главная часть разложения в ряд Лорана в окрестности изолированной особой точки $s=s_{j} \in \mathscr{Z}, j=1,2, \ldots, l$, аналитической функщии

$$
f(s) \stackrel{\text { def }}{=} \frac{1-\widehat{G}(s)}{a[1-\widehat{F}(s)]}
$$

и, аналогично, пусть $-B_{q} /(s-q)$ - главная часть разложения функции $f(s)$ в ряд Лорана в окрестности точки $s=q \in \mathscr{Z}$. Обозначим через $\mathscr{E}_{j}$ комплекснозначную меру с плотностью $\mathbf{I}_{(0, \infty)}(x) \exp \left(-s_{j} x\right)\left(\mathbf{I}_{A}(x)-\right.$ индикаторная функция множества $A)$; преобразование Лапласа этой меры равно $-1 /\left(s-s_{j}\right), \operatorname{Re}\left(s-s_{j}\right)<0$. Палее, пусть $\mathscr{E}_{q}-$ мера с плотностью $\mathbf{I}_{(0, \infty)}(x) \exp (-q x)$.

Теорема 1. Пусть $\left\{X_{k}\right\}_{k=1}^{\infty}-$ последовательность независимьх одинаково распределенных случайных величия с распределекием вероятностей $F$ и математическим ожидамием $\mu=\mathbf{E} X_{1} \in(-\infty, 0)$. Предположим, ито $\widehat{F}(r)<\infty$ и выполнены условия (а)-(в). Допустим, что $\widehat{F}(s) \neq 1$ ма прямой $\{s \in \mathrm{C}: \operatorname{Re} s=r\}$ u $\left(F^{m *}\right)_{s}(r)<1$ при некотором челом $m \geqslant 1$. Тогда дяя распределения $D$ супремума $M_{\infty}$ случайного блуждания $\left\{S_{n}\right\}$ справедливо предстаяление

$$
D=B_{q} \mathscr{E}_{q}+\sum_{j=1}^{l} \sum_{k=1}^{m_{j}} B_{j k} \mathscr{E}_{j}^{k *}+R
$$

где остатох $R$ удовлетворяет неравенству $\int_{0}^{\infty} \exp (r x)|R|(d x)<\infty$.

Д о к а з а т е л ь с т в о. Пусть $M=\sum_{j=1}^{l} m_{j}+2$. Рассмотрим функцию

$$
v(s) \stackrel{\text { def }}{=} \frac{[1-\widehat{F}(s)](s-r-1)^{M}}{s(s-q) \prod_{j=1}^{l}\left(s-s_{j}\right)^{m_{j}}}, \quad s \in \Pi(r) \backslash \mathscr{Z}, s \neq 0 .
$$

Доопределим значения функции $v(s)$ в точках $0, q, s_{j}, j=1, \ldots, l$, по непрерывности. Повторяя рассуждения из доказательства теоремы 1 в [16], устанавливаем, что функция $v(s)$ является преобразованием Лапласа $\widehat{V}(s)$ некоторой вещественнозначной меры $V \in \mathcal{S}_{r}$ и, более того, функция $w(s)=1 / v(s)$ - преобразование Лапласа $\widehat{W}(s)$ некоторой меры $W \in \mathcal{S}_{r}$. Положим $u(s) \stackrel{\text { def }}{=}[1-\widehat{G}(s)] w(s) / a$. Очевидно, что $u(s)-$ преобразование Лапласа $\widehat{U}(s)$ некоторой меры $U \in \mathfrak{G}_{r}$. Имеем

$$
\widehat{D}(s)=u(s) \frac{(s-r-1)^{M}}{s(s-q) \prod_{j=1}^{l}\left(s-s_{j}\right)^{m_{j}}}, \quad \text { Re } s=0 .
$$

Воспользуемся процедурой разложения рациональной функции на простые дроби. Получим

$$
\widehat{D}(s)=u(s)+\frac{A u(s)}{s}+\frac{B u(s)}{s-q}+\sum_{j=1}^{l} \sum_{k=1}^{m_{j}} \frac{b_{j k} u(s)}{\left(s-s_{j}\right)^{k}} .
$$

Преобразуем произвольное слагаемое в двойной сумме:

$$
\frac{b_{j k} u(s)}{\left(s-s_{j}\right)^{k}}=b_{j k} \sum_{i=0}^{k-1} \frac{u_{j, i}\left(s_{j}\right)}{\left(s-s_{j}\right)^{k-i}}+b_{j k} u_{j, k}(s)
$$

тде $u_{j, 0}(s) \stackrel{\text { def }}{=} u(s), u_{j, i}(s) \stackrel{\text { def }}{=}\left[u_{j, i-1}(s)-u_{j, i-1}\left(s_{j}\right)\right] /\left(s-s_{j}\right), i=1, \ldots, k$. Последовательно применяя теорему 2 из [7, приложение 2], устанавливаем принадлежность 
мер $U_{j, k}$ с преобразованиями Лапласа $\widehat{U}_{j, k}(s)=u_{j, k}(s), k=1, \ldots, m_{j}, j=1, \ldots, l, \mathrm{k}$ банаховой алгебре $\mathfrak{S}_{r}$. Наконец,

$$
\frac{B u(s)}{s-q}=\frac{B u(q)}{s-q}+\frac{B[u(s)-u(q)]}{s-q}=-\frac{B_{q}}{s-q}+B u_{q}(s),
$$

где по той же теореме $u_{q}(s)$ - преобразование Лапласа $\widehat{U}_{q}(s)$ некоторой меры $U_{q} \in$ $\mathfrak{S}_{r}$. Положим .

$$
R \stackrel{\text { def }}{=} U-\frac{A \cdot T(G) \cdot W}{a}+B \cdot U_{q}+\sum_{j=1}^{l} \sum_{k=1}^{m_{j}} b_{j k} U_{j, k} .
$$

В силу сказанного выше $R \in \mathcal{S}_{r}$. Подставляя (4) и (5) в (3) и приводя подобные члены, получим

$$
\widehat{D}(s)=-\frac{B_{q}}{s-q}+\sum_{j=1}^{l} \sum_{k=1}^{m_{j}}(-1)^{k} \frac{B_{j k}}{\left(s-s_{j}\right)^{k}}+\widehat{R}(s) .
$$

Тот факт, что в результате приведения подобных членов коэффициент при $\left(s-s_{j}\right)^{-k}$ будет в точности равен $(-1)^{k} B_{j k}$, следует из единственности разложения аналитической функции в ряд Лорана в окрестности изолированной особой точки. Для завершения доказательства теоремы 1 остается перейти от преобразований Лапласа $\mathrm{k}$ соответствуюшим мерам.

В качестве следствия теоремы 1 сформулируем утверждение об асимптотике вероятности $\mathbf{P}\left\{M_{\infty}>\boldsymbol{x}\right\}$.

Следствие. Пусть выполнены условия теоремы 1. Тогда

$$
\mathbf{P}\left\{M_{\infty}>x\right\}=\frac{B_{q} e^{-q x}}{q}+\sum_{j=1}^{I} \sum_{k=1}^{m_{j}} B_{j k} \mathscr{E}_{j}^{k *}((x, \infty))+R((x, \infty)),
$$

причем $|R((x, \infty))| \leqslant|R|((x, \infty))=o\left(e^{-r x}\right), x \rightarrow \infty$.

3 а м е ч н и е. Полученное в [11, теорема 2] асимптотическое разложение для вероятности $\mathbf{P}\left\{M_{\infty}>x\right\}$, учитывающее влияние корней характеристического уравнения, имеет следующий порядок убывания остатка: $R((x, \infty))=o\left(x^{m} e^{-r x}\right)$ при $x \rightarrow \infty$, где $m=\max _{1 \leqslant j \leqslant l} m_{j}-1$.

3. Осциллируюшее случайное блуждание.

О п р е д е ле н и е ([17]). Пусть $\left\{X_{n}\right\}_{n=0}^{\infty}$ и $\left\{Y_{n}\right\}_{n=0}^{\infty}$ - две независимые последовательности независимых одинаково распределенных случайных величин. ПІоследовательность случайных величин $\left\{Z_{n}\right\}_{n=0}^{\infty}$ называется осчиллируюиим случайным блужданием с управляющими последовательностями $\left\{X_{n}\right\}$ и $\left\{Y_{n}\right\}$, если задано начальное значение $Z_{0}$ и последующие значения $Z_{n}$ определяются рекуррентными соотношениями

$$
Z_{n+1}= \begin{cases}Z_{n}+X_{n}, & \text { если } Z_{n}>0 \\ Z_{n}+Y_{n}, & \text { если } Z_{n}<0, \\ Z_{n}+W_{n}, & \text { если } Z_{n}=0,\end{cases}
$$

где $W_{n}=X_{n}$ с вероятностью $p$ и $W_{n}=Y_{n}$ с вероятностью $1-p$.

Обозначим через $R_{X}$ и $R_{Y}$ множества возможных значений случайных величин $X_{1}$ и $Y_{1}$. Мы будем предполагать, что множество $R_{X} \cup R_{Y}$ - меарифметическое, т. е. что его элементы нельзя представить в виде $k d$, где $d-$ фиксированное положительное число, а $k \in \mathbf{Z}\left(\mathbf{Z}\right.$ - множество всех целых чисел). Пусть $F$ и $F^{*}-$ распределения случайных величин $X_{1}$ и $Y_{1}$ с конечными математическими ожиданиями $\mu=\mathbf{E} X_{1}<0$ и $\nu=\mathbf{E} Y_{1}>0$. В работе [18] получено явное выражение для 
стационарного распределения $\pi$ осциллирующего случайного блуждания $\left\{Z_{n}\right\}[18$, теорема 2].

В этом параграфе мы исследуем влияние корней характеристических уравнений $1-\widehat{F}(s)=0$ и $1-\widehat{F}^{*}(s)=0$ на свойства стационарного распределения $\pi$ осциллирующего случайного блуждания $\left\{Z_{n}\right\}$. С этой целью введем дополнительные обозначения.

Положим $S_{0}^{*}=0, S_{k}^{*}=\sum_{i=1}^{k} Y_{i}, k \geqslant 1$. Пусть $L_{\infty}^{*}=\inf \left\{S_{0}^{*}, S_{1}^{*}, \ldots\right\}$. Распределение случайной величины $L_{\infty}^{*}$ обозначим через $D^{*}$, а через $F_{-}$и $F_{+}^{*}$ обозначим соответственно распределения первой неположительной суммы $S_{N}$ случайного блуждания $\left\{S_{n}\right\}$ и первой неотрищательной суммы $S_{N^{*}}^{*}$ случайного блуждания $\left\{S_{n}^{*}\right\}$; здесь $N=\inf \left\{k \geqslant 1: S_{k} \leqslant 0\right\}$ и $N^{*}(x)=\inf \left\{k \geqslant 1: S_{k}^{*} \geqslant 0\right\}$.

Определим распределения вероятностей следующим образом: $H \stackrel{\text { def }}{=} T\left(F_{-}\right) / \mathrm{E} S_{N}$ и $H^{*} \stackrel{\text { def }}{=} T\left(F_{+}^{*}\right) / \mathbf{E} S_{N^{*}}^{*}$, где $T$ - omeратор, введенный в п. 1.

В этих обозначениях представление для стационарного распределения $\pi$ осциллирукшего случайного блуждания $\left\{Z_{n}\right\}$, полученное в работе [18], принимает следующий вид

$$
\pi=\frac{\nu}{\nu-\mu} D * H^{*}+\frac{-\mu}{\nu-\mu} D^{*} * H \stackrel{\text { def }}{=} \pi_{1}+\pi_{2} .
$$

Поскольку сужение $\left.\pi\right|_{(0, \infty)}$ меры $\pi$ на $(0, \infty)$ совпадает с $\pi_{1}$ (и, аналогично, $\left.\pi\right|_{(-\infty, 0)}=$ $\left.\pi_{2}\right)$, задача изучения влияния корней характеристических уравнений $1-\widehat{F}(s)=0$ и $1-\widehat{F}^{*}(s)=0$ на свойства стационарного распределения $\pi$ сводится к аналогичным задачам для мер $\pi_{1}$ и $\pi_{2}$.

Далее, наряду с $\mathscr{Z}$ рассмотрим множество $\Xi^{*}$ корней характеристического уравнения $1-\widehat{F}^{*}(s)=0$, лежаших в полосе $\Pi(r)$. Если $\Xi^{*}$ непусто, то его элементы обозначим через $\xi_{j}^{*}$, а их кратности - через $m_{j}^{*}, j=1, \ldots, l^{*}$. Допустим, что $\widehat{F}(r)$ и $\widehat{F}^{*}(r)$ конечны. Обозначим через $\sum_{k=1}^{n_{j}}(-1)^{k} C_{j k} /\left(s-s_{j}\right)^{k}$ главную часть разложения в ряд Лорана в окрестности изолированной особой точки $s=s_{j} \in \mathscr{Z}, j=1,2, \ldots, l$, аналитической функции

$$
f_{1}(s) \stackrel{\text { def }}{=} \frac{1-\widehat{G}(s)}{a[1-\widehat{F}(s)]} \widehat{H}^{*}(s),
$$

и аналогично $-C_{q} /(s-q)$ - главная часть разложения функции $f_{1}(s)$ в ряд Лорана в окрестности точки $q \in \mathscr{Z}$; здесь $n_{j}=m_{j}$, если $s_{j} \notin \Xi^{*}$, и $n_{j}=\max \left(0, m_{j}-m_{i}^{*}\right)$, если $s_{j}=\xi_{i}^{*} \in \Xi^{*}$. Отметим, что нули функции $\widehat{H}^{*}(s)$ в полосе $\Pi(r)$ совпадают с корнями характеристического уравнения $1-\widehat{F}^{*}(s)=0$, причем кратности нулей функцик $\widehat{H}^{*}(s)$ и соответствующих корней уравнения $1-\widehat{F}^{*}(s)=0$ одинаковы. Действительно, это вытекает из факторизационного тождества

$$
1-\widehat{F}^{*}(s)=\left[b^{*} \widehat{D}^{*}(s)\right]^{-1} \cdot\left[1-\widehat{F}_{+}^{*}(s)\right], \quad 0 \leqslant \operatorname{Re} s \leqslant r
$$

где $b^{*}=\exp \left\{\sum_{n=1}^{\infty} \mathbf{P}\left\{S_{n}^{*}<0\right\} / n\right\} ;$ тождество (8) является следствием теоремы о факторизации, леммы 2 из [1, гл. XVIII, § 3] и выражения для $\widehat{D}^{*}(s)$, симметричного представлению (1). Разделив обе части равенства (8) на. $-s$, получим

$$
\frac{\widehat{F}^{*}(s)-1}{s}=\left[b^{*} \widehat{D}^{*}(s)\right]^{-1} \mathbf{E} S_{N^{*}}^{*} \cdot \widehat{H}^{*}(s), \quad 0 \leqslant \operatorname{Re} s \leqslant r
$$

откуда немедленно вытекает утверждение о нулях функции $\widehat{H}^{*}(s)$.

Теорема 2. IIусть $\left\{Z_{n}\right\}_{n=0}^{\infty}-$ осциллируючее случаймое блуждание и $\pi$ его стачионарное распределение, определяемое по бормуле (7). Iредположим, ито выполнены условия (а)-(в) и $\widehat{F}^{*}(r)<\infty$. Допустим, что $\widehat{F}(s) \neq 1$ на прямой 
$\{s \in \mathbf{C :}: \operatorname{Re} s=r\}$ и $\left(F^{m *}\right)_{s}(r)<1$ при нехотором челом $m \geqslant 1$. Тогда для сужения $\left.\pi\right|_{(0, \infty)}$ стачионарного распределения $\pi$ на $(0, \infty)$ справедливо представление

$$
\left.\pi\right|_{(0, \infty)}=\frac{\nu}{\nu-\mu}\left[C_{q} \mathscr{E}_{q}+\sum_{j=1}^{l} \sum_{k=1}^{n_{j}} C_{j k} \mathscr{E}_{j}^{k *}+R_{1}\right],
$$

аде остаток $R_{1}$ удовлетворяет неравенству $\int_{0}^{\infty} \exp (r x)\left|R_{1}\right|(d x)<\infty$.

Д ок а з а т е л ь с т в о. Обозначим для краткости $h(s)=\hat{H}^{*}(s)$. Из соотношений (6) и (7) вытекает, что

$$
\widehat{\pi}_{1}(s)=\frac{\nu}{\nu-\mu}\left[-\frac{B_{q}}{s-q}+\sum_{j=1}^{l} \sum_{k=1}^{m_{j}} \frac{(-1)^{k} B_{j k}}{\left(s-s_{j}\right)^{k}}+\widehat{R}(s)\right] h(s) .
$$

Рассмотрим выражение $h(s) /\left(s-s_{j}\right)^{k}$. Используя уже применявшийся прием, получим

$$
\frac{h(s)}{\left(s-s_{j}\right)^{k}}=\sum_{i=0}^{k-1} \frac{h_{j, i}\left(s_{j}\right)}{\left(s-s_{j}\right)^{k-i}}+h_{j, k}(s),
$$

где $h_{j, 0}(s) \stackrel{\text { def }}{=} h(s), h_{j, i}(s) \stackrel{\text { def }}{=}\left[h_{j, i-1}(s)-h_{j, i-1}\left(s_{j}\right)\right] /\left(s-s_{j}\right), i=1, \ldots, k$. В силу теоремы 2 из [7, приложение 2] функции $h_{j, i}(s)$ суть преобразования Лапласа некоторых мер $H_{j, i} \in \mathfrak{S}_{r}$. Применяя указанную процедуру ко всем слагаемым двойной суммы и слагаемому $-B_{q} /(s-q)$ в правой части соотношения (10) и приводя подобные члены, получим соотношение (9), если $R_{1} \stackrel{\text { def }}{=} R * H^{*}+\sum_{j=1}^{l} \sum_{k=1}^{m_{j}}(-1)^{k} B_{j k} H_{j, k}-B_{q} H_{q} \in \mathfrak{S}_{r}$, где $H_{q} \in \mathfrak{S}_{r}-$ мера с преобразованием ЛІапласа $\hat{H}_{q}(s)=[h(s)-h(q)] /(s-q)$. Теорема доказана.

Картина на отрицательной полуоси выглядит совершенно симметричным образом. Поэтому мы сформулируем соответствующую теорему без доказательства. Предварительно введем однако еще несколько обозначений.

Пусть $r^{*}<0$ и $1<\widehat{F}^{*}\left(r^{*}\right)<\infty$. Обозначим $\Pi\left(r^{*}\right)=\left\{s \in \mathbf{C}: r^{*} \leqslant \operatorname{Re} s \leqslant\right.$ 0\} и $\mathscr{Z}^{*}=\left\{s \in \Pi\left(r^{*}\right): \widehat{F}^{*}(s)=1\right\} \backslash\{0\}$. Допустим, что $\widehat{F}^{*}(s) \neq 1$ на прямой $\left\{s \in \mathrm{C}: \operatorname{Re} s=r^{*}\right\}$ и преобразование Лапласа в точке $r^{*}$ сингулярной компоненты $\left[\left(F^{*}\right)^{m *}\right]_{s}$ свертки $\left(F^{*}\right)^{m *}$ меньше единицы: $\left[\left(F^{*}\right)^{m *}\right]_{s}\left(r^{*}\right)<1$. Тогда множество $\mathscr{Z}^{*}$ конечно и содержит единственный вещественный корень $q^{*}$ характеристического уравнения $1-\widehat{F}^{*}(s)=0$. Обозначим элементы множества $\mathscr{L}^{*}$ через $q^{*}, s_{1}^{*}, s_{2}^{*}, \ldots, s_{l^{*}}^{*}$, а кратность корня $s_{j}^{*}-$ через $m_{j}^{*}, j=1, \ldots, l^{*}$. Наряду с $\mathscr{Z}^{*}$ рассмотрим множество $\Xi$ корней характеристического уравнения $1-\widehat{F}(s)=0$, лежаших в полосе $\Pi\left(r^{*}\right)$. Если $\Xi$ непусто, то его элементы обозначим через $\xi_{j}$, а их кратности - через $m_{j}, j=$ $1, \ldots, l$ (оговоримся, что числа $m_{j}, m_{j}^{*}, l$ и $l^{*}$ необязательно те же, что в теореме 2 ). Допустим, что $\widehat{F}\left(r^{*}\right)<\infty$. Обозначим через $\sum_{k=1}^{n_{j}} D_{j k} /\left(s_{s}-s_{j}^{*}\right)^{k}$ главную часть разложения в ряд Лорана в окрестности изолированной особой точки $s=s_{j}^{*} \in \mathscr{L}^{*}$, $j=1,2, \ldots, l$, аналитической функции

$$
f_{2}(s) \stackrel{\text { def }}{=} \frac{1-\widehat{G}^{*}(s)}{a^{*}\left[1-\widehat{F}^{*}(s)\right]} \widehat{H}(s),
$$

где $G^{*}$ - распределение первой положительной суммы случчайного блуждания $\left\{S_{n}^{*}\right\}$ и $a^{*}=\exp \left\{\sum_{n=1}^{\infty} \mathbf{P}\left\{S_{n}^{*} \leqslant 0\right\} / n\right\} ;$ аналог ично, $D_{q} /\left(s-q^{*}\right)-$ главная часть разложения функции $f_{2}(s)$ в ряд Лорана в окрестности точки $q^{*} \in \mathscr{L}^{*}$. Кроме того, здесь $n_{j}=m_{j}$, если $s_{j}^{*} \notin \Xi$ и и $n_{j}=\max \left(0, m_{j}-m_{i}^{*}\right)$, если $s_{j}^{*}=\xi_{i} \in \Xi$. Нахонец, пусть $\mathscr{E}_{j}-$ мера с плотностью $\mathbf{I}_{(-\infty, 0)}(x) \exp \left(-s_{j}^{*} x\right), j=1, \ldots, l$, и $\underline{\mathscr{E}}_{q^{*}}-$ мера с плотностью $\mathbf{1}_{(-\infty, 0)}(x) \exp \left(-q^{*} x\right)$. 
Теорема 3. Iусть $\left\{Z_{n}\right\}_{n=0}^{\infty}$ - осчиллируюиее случайное блуждание $\boldsymbol{u} \pi$ его стачиояарное распределение, определяемое по формуле (7). Предположим, что выполмень условия (а)-(в), в которых символы $r, F$ и $\Pi(r)$ замемемь ма $r^{*}, F^{*} u$ $\Pi\left(r^{*}\right)$. Допустим, ито $\widehat{F}\left(r^{*}\right)<\infty, \widehat{F}^{*}(s) \neq 1$ на прямой $\left\{s \in\right.$ C: $\left.\operatorname{Re} s=r^{*}\right\} u$ $\left[\left(F^{*}\right)^{m *}\right]_{s}\left(r^{*}\right)<1$ при некотором челом $m \geqslant 1$. Тогда для сужения $\left.\pi\right|_{(-\infty, 0)}$ стаиионарного распределения $\pi$ на $(-\infty, 0)$ справедливо представление

$$
\left.\pi\right|_{(-\infty, 0)}=\frac{-\mu}{\nu-\mu}\left[D_{q} \mathscr{E}_{q^{*}}+\sum_{j=1}^{I} \sum_{k=1}^{n_{j}} D_{j k \mathscr{E}_{j}^{k *}}+R_{2}\right]
$$

где остаток $R_{2}$ удовлетворяет иеравенству $\int_{-\infty}^{0} \exp \left(r^{*} x\right)\left|R_{2}\right|(d x)<\infty$.

Из теорем 2 и 3 можно без труда получить асимптотические разложения с оценками остатков для величин $\pi((x, \infty)), x \rightarrow \infty$, и $\pi((-\infty, x]), x \rightarrow-\infty$ (см. следствие теоремы 1).

\section{СПИСОК ЛИТЕРАТУРЫ}

1. Феллер В. Введение в теорию вероятностей и ее приложения. Т. 2. М.: Наука, 1967, $752 \mathrm{c}$.

2. Kiefer $J$., Wolfowitz $J$. On the characteristics of the general queueing process, with applications to random walk. - Ann. Math. Statist., 1956, v. 27, p. 147-161.

3. Janson S. Moments for first passage times, the minimum, and related quantities for random walks with positive drift. - Adv. Appl. Probab., 1986, v. 18, p. 865-879.

4. Tweedie R. L. The existence of moments for stationary Markov chains. - J. Appl. Probab., 1983, v. 20, p. 191-196.

5. Alsmeyer $G$. On generalized renewal measures and certain first passage times. Ann. Probab., 1992, v. 20, p. 1229-1247.

6. Sgibnev M. S. Submultiplicative moments of the supremum of a random walk with negative drift. - Statist. Probab. Lett., 1997, v. 32, p. 377-383.

7. Боровков A. A. Вероятностные процессы в теории массового обслуживания. М.: Наука, 1972, $368 \mathrm{c.}$

8. Veraverbeke $N$. Asymptotic behaviour of Wiener-Hopf factors of a random walk. Stochastic Process. Appl., 1977, v. 5, p. 27-37.

9. Сеибнев $M$. C. О распределении супремума сумм независимых слагаемых с отрицательным сносом. - Матем. заметки, 1977, т. 22, № 5, с. 763-770.

10. Embrechts $P$., Veraverbeke $N$. Estimates for the probability of ruin with special emphasis on the possibility of large claims. - Insurance: Mathematics and Economics, 1982 , v. 1, p. 55-72.

11. Лотов В. И. Асимптотика распределения супремума последовательных сумм. Матем. заметки, 1985 , т. 38 , № 5, с. 668-678.

12. Bertoin J., Doney R. A. Some asymptotic results for transient random walks. J. Appl. Probab., 1996, v. 28, p. 206-226.

13. Боровков A. A., Коричмов Д. А. Вероятности больших уклонений для одномерных цепей Маркова. I. Стационарные распределения. - Теория вероятн. и ее примен., 1996, т. 41, в. 1, с. 3-30.

14. Van der Genugten B. B. Asymptotic expansions in renewal theory. - Compos. Math., 1969, v. 21, № 4, p. 331-342.

15. Хилле Э., Филлипс Р. Функциональный анализ и полугрупाы. М.: ИЛ, 1962.

16. Саибнев $М$. С. Экспоненциальные оценки скорости сходимости для старших моментов восстановления. - Сиб. матем. журн., 1991, т. 32, № 4, с. 143-152.

17. Kemperman J. H. B. The oscillating random walk. - Stochastic Process. Appl., 1974 , v. 2, p. 1-29. 
18. Боровков A. A. Предельное распределение для осциллирующего случайного блуждания. - Теория вероятн. и ее примен., 1980, т. XXV, в. 3, с. 663-665.

Поступила в редакцию 5.XII. 1997

$1998 \mathrm{r}$.

СЕВАСТЬЯНОВ Б. А.*

\section{АСИМПТОТИЧЕСКОЕ ПОВЕДЕНИЕ ВЕРОЯТНОСТЕЙ ВЫРОЖДЕНИЯ ОСТАНОВЛЕННЫХ ВЕТВЯЩИХСЯ ПРОЩЕССОВ ${ }^{1)}$}

Первоначальный многотипный ветвящийся процесс Гальтона-Ватсона

$$
\mu(t)=\left(\mu_{1}(t), \mu_{2}(t), \ldots, \mu_{m}(t)\right), \quad t=0,1,2, \ldots,
$$

порождает остановленный ветвяшийся процесс $\xi(t)$, если при попадании $\mu(t)$ в некоторое конечное множество $S$ процесс останавливается. Предполагается, что первоначальный ветвящийся прошесс $\mu(t)$ докритичен и неразложим. Доказано, что вероятность вырождения

$$
q_{r}^{n}=\lim _{t \rightarrow \infty} P\{\xi(t)=r \mid \xi(0)=n\}
$$

для любых $r=\left(r_{1}, r_{2}, \ldots, r_{m}\right) \in S, n=\left(n_{1}, \ldots, n_{m}\right) \notin S$ при $\bar{n}=n_{1}+\cdots+n_{m} \rightarrow$ $\infty, n_{i} / \bar{n} \rightarrow a_{i}$ асимптотически сближается с периодической с периодом 1 функцией от $\log _{1 / R} \bar{n}$, где $R<1$ - перронов корень матрицы математических ожиданий $A_{i j}=\mathbf{E}\left\{\mu_{j}(1) \mid \mu(0)=e(i)\right\}$ первоначального докритического ветвяшегося процесса $\mu(t)=\left(\mu_{1}(t), \mu_{2}(t), \ldots, \mu_{m}(t)\right)$, а вектор $e(i)=\left(\delta_{i 1}, \delta_{i 2}, \ldots, \delta_{i m}\right)$, $\delta_{i j}$ символ Кронекера.

Клячевые слова $и$ фразы: многотипный ветвящийся процесс Гальтона-Ватсона, неразложимый ветвящийся проџесс, докритический ветвящийся процесс, остановленный ветвящийся процесс. вероятности вырождения.

1. Постановка задачи. В работе рассматривается остановленный ветвяцийся процесс с несколькими типами частиц, который является некоторой модификацией многотипного ветвящегося процесса Гальтона-Ватсона. Обозначим $\mathbf{N}_{0}=\{0,1,2, \ldots\}$ множество неотрицательных целых чисел. Буквами $n, k, r, \mu, \xi$ и др. будем обозначать $m$-мерные векторы. Обозначение 0 будет использоваться и как $\boldsymbol{m}$-мерный вектор, и как числовой нуль. В дальнейшем будем также обозначать $a^{b}=a_{1}^{b_{1}} a_{2}^{b_{2}} \cdots a_{m}^{b_{m}}$, $a !=a_{1} ! a_{2} ! \cdots a_{m} !$, где $a=\left(a_{1}, \ldots, a_{m}\right), b=\left(b_{1}, \ldots, b_{m}\right)$, и $e(j)=\left(\delta_{j 1}, \ldots, \delta_{j m}\right)$, $\delta_{i j}-$ символ Кронекера. Будем также пользоваться обозначением $\delta_{\alpha \beta}=\prod_{i=1}^{m} \delta_{a_{i} \beta_{i}}$, $\alpha, \beta \in \mathbf{N}_{0}^{m}$.

Положим $\mu(t)=\left(\mu_{1}(t), \ldots, \mu_{m}(t)\right), \mu_{i}(t) \in \mathbf{N}_{0}$, где $\mu_{i}(t)$ - число частиц типа $T_{i}$ в $t$-м поколении ветвящегося процесса с типами частиц $T_{1}, T_{2}, \ldots, T_{m}$ и с дискретным временем $t=0,1,2, \ldots$. Производящие функции этого процесса

$$
h_{i}\left(s_{1}, s_{2}, \ldots, s_{m}\right)=h_{i}(s)=\mathbf{E}\left\{s^{\mu(1)} \mid \mu(0)=e(i)\right\}
$$

* Математический институт им. В. А. Стеклова, ул. Губкина, 8, 117966 Москва, ГСПI-1, Россия.

1) Работа выполнена при финансовой поддержке Российского фонда фундаментальных исследований, гранты 96-01-00338, 96-15-96092, и INTAS-RFBR 95-0099. 